\title{
Die Eigensprache der Kinder
}

\section{Wilhelm Felder}

Prof. em. Dr. med., Facharzt für Kinder- und Jugendpsychiatrie und-psychotherapie, Mitglied FMH

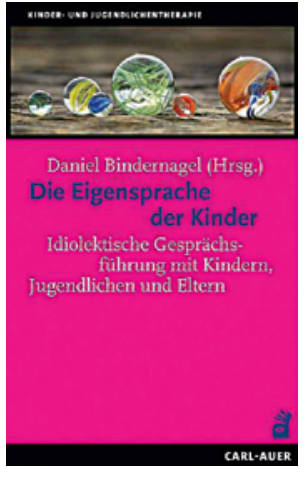

Daniel Bindernagel (Hrsg.)

Die Eigensprache der Kinder

Idiolektische Gesprächsführung mit Kindern,

Jugendlichen und Eltern

Heidelberg: Carl-Auerverlag; 2016.

279 Seiten, 46.50 CHF.

ISBN 978-3-8497-0054-6

Daniel Bindernagel ist Facharzt für Kinder- und Jugendpsychiatrie und -psychotherapie. Das anzuzeigende Buch ist sein zweites zum Thema. Der Untertitel des Buches heisst: Idiolektische Gesprächsführung mit Kinder, Jugendlichen und Eltern. Wikipedia sei Dank, können wir uns schnell orientieren, was idiolektische Gesprächsführung ist: «Unter dem Idiolekt versteht man das individuelle Sprachmuster eines Sprechenden mit all seinen phonetischen, grammatikalischen und die Wortwahl betreffenden Vorlieben.»

Im ersten Kapitel gibt Daniel Bindernagel einen Überblick über die Entwicklung der Eigensprache. Nach dem einleitenden Kapitel werden die verschiedenen Entwicklungsstadien im Einzelnen beschrieben: Babyund Kleinkindalter, Kleinkind, Schulalter, Jugendalter. Wie im Untertitel angekündigt, beschäftigt sich ein Kapitel auch mit der Eigensprache von Eltern. In einem abschliessenden Kapitel wird auf die Eigensprache von Fachpersonen in ihrer Bedeutung für die interdisziplinäre Zusammenarbeit hingewiesen.

Unser Wissen, wie sich Kinder entwickeln, ist schon recht umfangreich. Wollen wir uns näher damit beschäftigen, landen wir ganz schnell bei Buchkapiteln und Büchern über Teilaspekte: Sprachentwicklung, kognitive Entwicklung, emotionale Entwicklung, kultur- und schichtspezifische Aspekte der Entwicklung.
Die Leistung von Daniel Bindernagel, der sechs der acht Kapitel allein oder mit Koautoren geschrieben hat, liegt in der Integration dieser Teilaspekte. Er zeigt, wie die Sprache eines Menschen eben viel mehr ist, als sich aus der Addition von Alter, Geschlecht, Intelligenz, Kultur und Schicht ergibt. «Wir können Sprache also als die interaktive Oberfläche des Menschen betrachten, über die er mit seiner Umgebung in Kontakt tritt» (S. 24). Hier wie im ganzen Buch geht es dem Autor darum, zu zeigen, wie wichtig es für Therapeuten, Lehrer, Ärzte, Eltern ist, die Besonderheiten der Sprache jedes Menschen zu beachten, um mit diesem in eine gute Interaktion zu kommen. Diese gute Interaktion ist nicht nur die effiziente Interaktion als Mittel zum Zweck, damit das Kind schneller lernt, besser gehorcht. Je besser wir die Eigensprache unseres Gegenübers erfassen, umso persönlicher ist die Begegnung mit ihm. Im ganzen Buch wird spürbar, wie sehr es dem Autor eben nicht nur um die Vermittlung einer möglichst cleveren Sozialtechnik geht, sondern um die Vermittlung eines Menschenbildes. Diesem Menschenbild begegnen wir etwa in den Ausführungen zur Entwicklung: «Das Schulkind lernt das logische Denken in den Regeln der Schrift, der Grammatik, der Sprache, der Natur, der Mathematik und der sozialen Gemeinschaft ... Das 'paralogische' Denken braucht eine offene Aufmerksamkeit mit achtsamer Wahrnehmung und Beobachtung von Sinneswahrnehmungen im Hier und Jetzt. Hier geht es um Loslassen, darum, den Geist denken zu lassen, was er will. Hier werden Bilder, Geschichten und Metaphern geboren und weitergesponnen. Im besten Falle lernt das Schulkind, beide Denkarten zu nutzen» (S. 61). Dieses Menschenbild beinhaltet auch die Überzeugung, dass das Gegenüber, der Patient, nicht nur über Ressourcen verfügt, sondern auch über die Fähigkeit, die Lösung für seine Probleme zu finden, wenn er denn dazu die nötige Unterstützung bekommt. Im Kapitel über die Eigensprache der Eltern, das der Autor mit einem Hausarzt geschrieben hat, werden zahlreiche Beispiele von körperlich und psychisch kranken Eltern dargestellt. Die idiolektische Gesprächsführung wird anhand dieser Beispiele anschaulich beschrieben.

Das Buch eignet sich dank der verständlichen Sprache, in der es geschrieben ist, für ein breites Publikum von Fachpersonen, aber auch für interessierte Laien. Kritik am Buch würde ich äussern, wenn es etwas zu kritisieren gäbe. Ich habe jedoch keine Schwachstellen gefunden. 